\title{
Resummation with Wilson lines off the light cone
}

\author{
Hsiang-nan $\mathrm{Li}$ 米 \\ ${ }^{1}$ Institute of Physics, Academia Sinica, Taipei, Taiwan 115, Republic of China, \\ ${ }^{1}$ Department of Physics, Tsing-Hua University, \\ Hsinchu, Taiwan 300, Republic of China, and \\ ${ }^{1}$ Department of Physics, National Cheng-Kung University, Tainan, Taiwan 701, Republic of China
}

\begin{abstract}
I review the resummation formalism for organizing large logarithms in perturbative expansion of collinear subprocesses through the variation of Wilson lines off the light cone. A master equation is derived, which involves the evolution kernel resulting from this variation. It is then demonstrated that all the known single- and double-logarithm summations for a parton distribution function or a transverse-momentum-dependent parton distribution can be reproduced from the master equation by applying appropriate soft-gluon approximations to the evolution kernel. Moreover, jet substructures, information which is crucial for particle identification at the Large Hadron Collider and usually acquired from event generators, can also be calculated in this formalism.
\end{abstract}

PACS numbers:

\section{INTRODUCTION}

It is known that radiative corrections in perturbative QCD (pQCD) produce large logarithms at each order of the coupling constant. Double logarithms appear in processes involving two scales, such as $\ln ^{2}\left(p^{+} b\right)$ with $p^{+}$ being the large longitudinal momentum of a parton and $b$ being the impact parameter conjugate to the small parton transverse momentum $k_{T}$. In the region with a large Bjorken variable $x$, there exists $\ln ^{2}(1 / N)$ from the Mellin transformation of $\ln (1-x) /(1-x)_{+}$, for which the two scales are the large $p^{+}$and the small infrared cutoff $(1-x) p^{+}$for gluon emissions from a parton. Single logarithms are generated in processes involving one scale, such as $\ln p^{+}$and $\ln (1 / x)$, for which the relevant scales are the large $p^{+}$and the small $x p^{+}$, respectively. To improve perturbative expansion, these logarithmic corrections need to be organized by evolution equations or resummation techniques. Various methods have been developed to organize these logarithmic corrections to a parton distribution function (PDF) or to a transverse-momentum-dependent distribution function (TMD): the $k_{T}$ resummation for $\ln ^{2}\left(p^{+} b\right)$ [1, 2], the threshold resummation for $\ln ^{2}(1 / N)$ [3 [5], the joint resummation 6, 7] that unifies the above two formalisms, the Dokshitzer-Gribov-Lipatov-Altarelli-Parisi (DGLAP) equation for $\ln p^{+}[8]$, the Balitsky-Fadin-Kuraev-Lipatov (BFKL) equation for $\ln (1 / x)$ [9], and the Ciafaloni-CataniFiorani-Marchesini (CCFM) equation [10] that combines the above two evolution equations.

The definition of a PDF or a TMD contains Wilson lines along the light cone, which collect gluons collimated to a beam particle of momentum $p$ and attaching to other parts of a scattering process. The Wilson lines contain vertical links at infinity, if a TMD is considered. To perform resummation, a simple trick is to vary the Wilson lines off the light cone into an arbitrary direction $n^{\mu}$ with $n^{2} \neq 0$ [11]. The PDF or the TMD must depend on $p^{\mu}$ and $n^{\mu}$ through the Lorentz invariants $p \cdot n$ and $n^{2}$. When a parton $k_{T}$ is involved, the phase space of real radiation is constrained, so the associated infrared enhancement does not cancel completely that in virtual correction. The infrared enhancement then generates the double logarithms of the ratio $(p \cdot n)^{2} /\left(k_{T}^{2} n^{2}\right)$, and the variation of $n$ turns into the variation of the scale $p^{+}$or $k_{T}$. The key is that all different choices of the vector $n$ are equivalent in the viewpoint of collecting the collinear divergences associated with the beam particle. Therefore, the effect from varying $n$ does not involve the collinear divergences, which can then be factorized out of the PDF or the TMD, leading to an evolution equation in $n$. The resummation technique via the variation of the Wilson lines off the light cone will be reviewed in this article, and its wide applications to the single- and double-logarithmic summations will be demonstrated.

It has been a long-standing challenge to predict substructures (including masses and energy profiles) of lightquark and gluon jets in the PQCD theory: fixed-order QCD calculations cannot describe experimental data on

*Electronic address: hnli@phys.sinica.edu.tw 


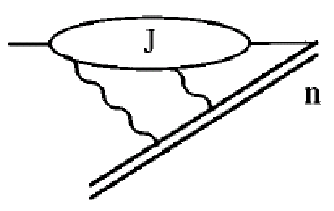

(a)

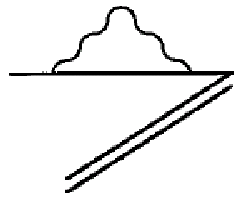

(b)

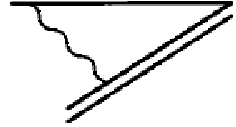

(c)

FIG. 1: (a) Jet subprocess defined in Eq. (1). (b) and (c) LO diagrams of (a).

jet substructures, especially in extreme kinematic regions, such as the region with a small jet invariant mass. Hence, it is a custom for experimentalists to compare measured jet substructures with predictions from full event generators such as PYTHIA or HERWIG. While the full event generators (usually with specific tuning) could describe data, it remains desirable to develop a theoretical framework for the study of jet substructures. A novel approach to predicting jet substructures based on the resummation formalism was proposed recently 12. It has been shown that results of this formalism for light-quark and gluon jets are well consistent with the mass distributions measured by CDF [13], and with the energy profiles measured by CDF at Tevatron [14] and CMS at Large Hadron Collider (LHC) [15].

\section{RESUMMATION FORMALISM}

In this section I explain the basic idea of the resummation formalism with the Wilson lines off the light cone. Collinear and soft divergences in perturbation theory may overlap to form double logarithms in extreme kinematic regions with low $p_{T}$ and large $x$. The former includes low $p_{T}$ jet, photon, and $W$ boson productions, which all require real gluon emissions of small $p_{T}$. The latter includes top pair production, deeply inelastic scattering (DIS), Drell-Yan production, and heavy meson decays $B \rightarrow X_{u} l \nu$ and $B \rightarrow X_{s} \gamma$ [16 18] at the end points, for which parton momenta remain large, and radiations are constrained in the soft region. Because of the limited phase space for real corrections, the infrared cancellation is not complete. The double logarithms, appearing in products with the coupling constant $\alpha_{s}$, such as $\alpha_{s} \ln ^{2}\left(E / p_{T}\right)$ with the beam energy $E$ and $\alpha_{s} \ln (1-$ $x) /(1-x)_{+}$, deteriorate perturbative expansion. Double logarithms also occur in exclusive processes, such as Landshoff scattering [19], hadron form factors [20], Compton scattering [21] and heavy-to-light transitions $B \rightarrow \pi(\rho)$ [22] and $B \rightarrow D^{(*)}$ [23] at maximal recoil. In order to have a reliable pQCD analysis of these processes, the important logarithms need to be summed to all orders.

Take as an example a jet subprocess defined by the matrix element in the covariant gauge $\partial \cdot A=0$ [18],

$$
J(p, n) u(p)=\left\langle 0\left|\mathcal{P} \exp \left[-i g \int_{0}^{\infty} d z n \cdot A(n z)\right] q(0)\right| p\right\rangle,
$$

where $q$ is a light quark field with momentum $p, u(p)$ is a spinor, and $A$ is a gluon field. The abelian case of this subprocess has been discussed in 24]. The path-ordered exponential in Eq. (1) is the consequence of the factorization of collinear gluons with momenta parallel to $p$ from a full process. For convenience, it is assumed that $p$ has a large light-cone component $p^{+}$, and all its other components vanish. A general diagram of the jet function $J$ is shown in Fig. 1(a), where the path-ordered exponential is represented by a double line along the arbitrary vector $n$. As stated before, varying the direction $n$ does not change the collinear divergences collected by the Wilson line.

It is easy to see that $J$ contains double logarithms from the overlap of collinear and soft divergences by calculating the leading-order (LO) diagrams in Fig. 1(b), the self-energy correction, and in Fig. 1(c), the vertex correction. In the covariant gauge Fig. 1(b) (Fig. 1(c)) produces a single (double) logarithm. In the axial gauge $n \cdot A=0$ the path-ordered exponential reduces to an identity, and Fig. 1(c) does not exist. The essential step in the resummation technique is to derive a differential equation $p^{+} d J / d p^{+}=C J$ [18], where the coefficient function $C$ contains only single logarithms, and can be treated by renormalization-group (RG) methods. Since the path-ordered exponential is scale-invariant in $n, J$ must depend on $p$ and $n$ through the ratio $(p \cdot n)^{2} / n^{2}$. 


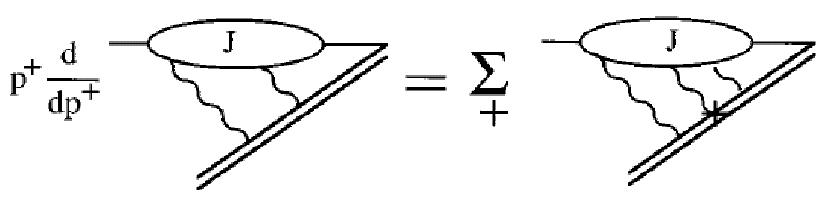

FIG. 2: Derivative $p^{+} d J / d p^{+}$in the covariant gauge.

The differential operator $d / d p^{+}$can then be replaced by $d / d n$ using a chain rule

$$
p^{+} \frac{d}{d p^{+}} J=-\frac{n^{2}}{v \cdot n} v_{\alpha} \frac{d}{d n_{\alpha}} J,
$$

with the vector $v=\left(1,0, \mathbf{0}_{T}\right)$ being defined via $p=p^{+} v$.

Equation (2) simplifies the analysis tremendously, because $n$ appears only in the Feynman rules for the Wilson line, while $p$ may flow through the whole diagram in Fig. 1(a). The differentiation of each eikonal vertex and of the associated eikonal propagator with respect to $n_{\alpha}$,

$$
-\frac{n^{2}}{v \cdot n} v_{\alpha} \frac{d}{d n_{\alpha}} \frac{n_{\mu}}{n \cdot l}=\frac{n^{2}}{v \cdot n}\left(\frac{v \cdot l}{n \cdot l} n_{\mu}-v_{\mu}\right) \frac{1}{n \cdot l} \equiv \frac{\hat{n}_{\mu}}{n \cdot l},
$$

leads to the special vertex $\hat{n}_{\mu}$. The derivative $p^{+} d J / d p^{+}$is thus expressed as a summation over different attachments of $\hat{n}_{\mu}$, labeled by the symbol + in Fig. 2. If the loop momentum $l$ is parallel to $p$, the factor $v \cdot l$ vanishes, and $\hat{n}_{\mu}$ is proportional to $v_{\mu}$. When this $\hat{n}_{\mu}$ is contracted with a vertex in $J$, in which all momenta are mainly parallel to $p$, the contribution to $p^{+} d J / d p^{+}$is suppressed. Hence, the leading regions of $l$ are soft and hard.

According to this observation, we investigate some two-loop examples exhibited in Fig. 3(a). Note that the third and fourth diagrams in Fig. 3(a), involving the crossing gluons, do not mean three-loop diagrams. If the loop momentum flowing through the special vertex is soft but another is not, only the first diagram is important, giving a large single logarithm. In this soft region the subdiagram containing the special vertex can be factorized using the eikonal approximation as shown in Fig. 3(b), where the symbol $\otimes$ represents a convoluting relation. The subdiagram is absorbed into a soft kernel $K$, and the remainder is identified as the original jet function $J$, both being $O\left(\alpha_{s}\right)$ contributions. If both the loop momenta are soft, the four diagrams in Fig. 3(a) are equally important. The subdiagrams, factorized according to Fig. 3(c), contribute to $K$ at $O\left(\alpha_{s}^{2}\right)$, and the remainder is the LO diagram of $J$. If the loop momentum flowing through the special vertex is hard and another is not, the second diagram in Fig. 3(a) dominates. In this region the subdiagram containing the special vertex is factorized as shown in Fig. 3(d). The right-hand side of the dashed line is absorbed into a hard kernel $G$ as an $O\left(\alpha_{s}\right)$ contribution, and the left-hand side is identified as the $O\left(\alpha_{s}\right)$ diagram of $J$. If both the loop momenta are hard, all the diagrams in Fig. 3(a) are absorbed into $G$, giving the $O\left(\alpha_{s}^{2}\right)$ contributions.

Extending the above reasoning to all orders, one derives the differential equation

$$
p^{+} \frac{d}{d p^{+}} J=\left[K\left(m / \mu, \alpha_{s}(\mu)\right)+G\left(p^{+} \nu / \mu, \alpha_{s}(\mu)\right)\right] J,
$$

where the coefficient function $C$ has been written as the sum of the soft kernel $K$ and the hard kernel $G$. In the above expression $\mu$ is a factorization scale, the gauge factor in $G$ is defined as $\nu=\sqrt{(v \cdot n)^{2} /\left|n^{2}\right|}$, and a gluon mass $m$ has been introduced to regularize the infrared divergence in $K$. Note that the function $J$ defined in Eq. (11) is not a physical object, and is not infrared safe, so the infrared regulator $m$ is needed [24]. For physical objects, such as the TMD discussed in the next section, this infrared regulator is not necessary. The $O\left(\alpha_{s}\right)$ contribution to $K$ from Fig. 3(b) is written as

$$
K=-i g^{2} C_{F} \mu^{\epsilon} \int \frac{d^{4-\epsilon} l}{(2 \pi)^{4-\epsilon}} \frac{\hat{n}_{\mu}}{n \cdot l} \frac{g^{\mu \nu}}{l^{2}-m^{2}} \frac{v_{\nu}}{v \cdot l}-\delta K,
$$

where

$$
\delta K=-\frac{\alpha_{s}}{2 \pi} C_{F}\left(\frac{2}{\epsilon}+\ln 4 \pi-\gamma_{E}\right)
$$




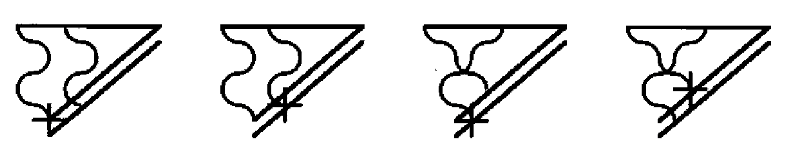

(a)

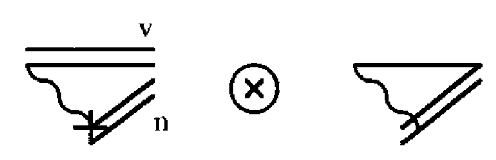

(b)

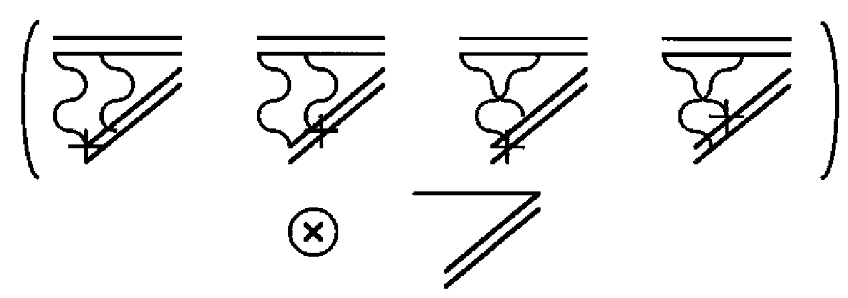

(c)

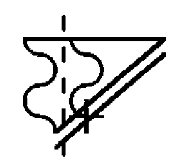

(d)

FIG. 3: (a) $O\left(\alpha_{s}^{2}\right)$ examples for the differentiated $J$. (b) Factorization of $K$ at $O\left(\alpha_{s}\right)$. (c) Factorization of $K$ at $O\left(\alpha_{s}^{2}\right)$. (d) Factorization of $G$ at $O\left(\alpha_{s}\right)$.

is an additive counterterm in the $\overline{\mathrm{MS}}$ scheme, $\gamma_{E}$ being the Euler constant. The $O\left(\alpha_{s}\right)$ contribution to $G$ from Fig. [3(d) is given by

$$
G=-i g^{2} C_{F} \mu^{\epsilon} \int \frac{d^{4-\epsilon} l}{(2 \pi)^{4-\epsilon}} \frac{\hat{n}_{\mu}}{n \cdot l} \frac{g^{\mu \nu}}{l^{2}}\left(\frac{\not p+\not l}{(p+l)^{2}} \gamma_{\nu}-\frac{v_{\nu}}{v \cdot l}\right)-\delta G,
$$

where the second term in the parentheses acts as a soft subtraction to avoid double counting, and $\delta G$ is an additive counterterm. Because of the soft cancellation between the two terms in the above expression, the infrared regulator $m$ has been dropped. A straightforward evaluation shows that Eqs. (5) and (6) contain only the single logarithms $\ln (m / \mu)$ and $\ln \left(p^{+} \nu / \mu\right)$, respectively, as expected. Organizing these single logarithms using RG methods, and then solving Eq. (4), one resums the double logarithms $\ln ^{2}\left(p^{+} / m\right)$ in $J$.

To reproduce all the known resummations and evolution equations, we construct a master equation for the $\operatorname{TMD} \Phi\left(x, k_{T}\right)$ following the above procedures. The dependence on a factorization scale $\mu$ is implicit. The factorization scale is similar to a renormalization scale, but introduced in perturbative computations for an effective theory. If the parton is a quark, $\Phi$ is defined by

$$
\begin{aligned}
\Phi_{q / N}\left(x, k_{T}\right)= & \int \frac{d y^{-}}{2 \pi} \int \frac{d^{2} y_{T}}{(2 \pi)^{2}} e^{-i x p^{+} y^{-}+i \mathbf{k}_{T} \cdot \mathbf{y}_{T}} \\
& \times \frac{1}{2}\left\langle N(p, \sigma)\left|\bar{q}\left(0, y^{-}, \mathbf{y}_{T}\right) \frac{1}{2} \gamma^{+} W\left(y^{-}, \mathbf{y}_{T}, 0,0_{T}\right) q\left(0,0,0_{T}\right)\right| N(p, \sigma)\right\rangle,
\end{aligned}
$$

where $|N(p, \sigma)\rangle$ denotes the bound state of the nucleon with momentum $p$ and spin $\sigma, y=\left(0, y^{-}, \mathbf{y}_{T}\right)$ is the coordinate of the quark field after the final-state cut, the first factor $1 / 2$ is attributed to the average over the nucleon spin, and the matrix $\gamma^{+} / 2$ is the spin projector for the nucleon. The Wilson links are defined as 
$W\left(y^{-}, \mathbf{y}_{T}, 0,0_{T}\right)=W\left(0,0_{T}\right) I_{0, \mathbf{y}_{T}} W^{\dagger}\left(y^{-}, \mathbf{y}_{T}\right)$ with the vertical link $I_{0, \mathbf{y}_{T}}$ being located at $y^{-}=\infty$ [25], and

$$
W\left(y^{-}, \mathbf{y}_{T}\right)=\mathcal{P} \exp \left[-i g \int_{0}^{\infty} d z n \cdot A(y+z n)\right] .
$$

The two quark fields before and after the final-state cut in Eq. (7) are separated by a distance, so the above Wilson links are demanded by the gauge invariance of the TMD as a nonlocal matrix element. More investigations on the vertical Wilson links can be found in [26]. If the parton is a gluon, the nonlocal operator in Eq. (77) is replaced by $F_{\mu}^{+}\left(0, y^{-}, y_{T}\right) F^{\mu+}\left(0,0,0_{T}\right)$.

Similarly, $n$ is varied arbitrarily away from the light cone with $n^{2} \neq 0$. Then $\Phi$ depends on $p^{+}$via the ratio $(p \cdot n)^{2} / n^{2}$, so the chain rule in Eq. (2) relating the derivative $d \Phi / d p^{+}$to $d \Phi / d n_{\alpha}$ applies. One derives the master equation

$$
p^{+} \frac{d}{d p^{+}} \Phi\left(x, k_{T}\right)=2 \bar{\Phi}\left(x, k_{T}\right),
$$

where $\bar{\Phi}$ contains the special vertex, and the coefficient 2 is due to the equality of $\bar{\Phi}$ with the special vertex on either side of the final-state cut. The function $\bar{\Phi}$ is factorized into the convolution of the soft and hard kernels with $\Phi$ :

$$
\bar{\Phi}\left(x, k_{T}\right)=\bar{\Phi}_{s}\left(x, k_{T}\right)+\bar{\Phi}_{h}\left(x, k_{T}\right)
$$

with the soft contribution

$$
\begin{aligned}
\bar{\Phi}_{s}= & {\left[-i g^{2} C_{F} \mu^{\epsilon} \int \frac{d^{4-\epsilon} l}{(2 \pi)^{4-\epsilon}} \frac{\hat{n} \cdot v}{n \cdot l l^{2} v \cdot l}-\delta K\right] \Phi\left(x, k_{T}\right) } \\
& -i g^{2} C_{F} \mu^{\epsilon} \int \frac{d^{4-\epsilon} l}{(2 \pi)^{4-\epsilon}} \frac{\hat{n} \cdot v}{n \cdot l v \cdot l} 2 \pi i \delta\left(l^{2}\right) \Phi\left(x+l^{+} / p^{+},\left|\mathbf{k}_{T}+\mathbf{l}_{T}\right|\right),
\end{aligned}
$$

where the first term is the same as in Eq. (5), and the second term proportional to $\delta\left(l^{2}\right)$ arises from the real soft gluon emission. The hard contribution is given by $\bar{\Phi}_{h}\left(x, k_{T}\right)=G\left(x p^{+} \nu / \mu, \alpha_{s}(\mu)\right) \Phi\left(x, k_{T}\right)$, in which the hard kernel $G$ is the same as in Eq. (6).

\section{III. $k_{T}$ RESUMMATION AND BFKL EQUATION}

The TMD definition in Eq. (77) contains three scales: $(1-x) p^{+}, x p^{+}$, and $k_{T}$. We first consider the soft approximation corresponding to the rapidity ordering of real gluon emissions in a ladder diagram. Assume that a parton carries the longitudinal momentum $x p^{+}+l_{2}^{+}+l_{1}^{+}$, which becomes $x p^{+}+l_{1}^{+}$after emitting a gluon of longitudinal momentum $l_{2}^{+}$and transverse momentum $l_{2 T}$, and then becomes $x p^{+}$after emitting a gluon of longitudinal momentum $l_{1}^{+}$and transverse momentum $l_{1 T}$. In the kinematic configuration with $l_{2}^{+} \gg l_{1}^{+}$and $l_{2 T} \sim l_{1 T}$, the original parton momentum is approximated by $x p^{+}+l_{2}^{+}+l_{1}^{+} \approx x p^{+}+l_{2}^{+}$. The loop integral associated with the first gluon emission is then independent of $l_{1}^{+}$, and can be worked out straightforwardly, giving a logarithm. The loop integral associated with the second gluon emission, involving only $l_{1}^{+}$, also gives a logarithm. Hence, a ladder diagram with $N$ rung gluons generates the logarithmic correction $\left(\alpha_{s} L\right)^{N}$ under the above ordering, where $L$ denotes the large logarithm. Following the rapidity ordering, we adopt the approximation for the real gluon emission in Eq. (11)

$$
\Phi\left(x+l^{+} / p^{+},\left|\mathbf{k}_{\mathbf{T}}+\mathbf{l}_{\mathbf{T}}\right|\right) \approx \Phi\left(x,\left|\mathbf{k}_{\mathbf{T}}+\mathbf{l}_{\mathbf{T}}\right|\right),
$$

where the $l^{+}$dependence has been neglected. The transverse momenta $l_{T}$, being of the same order as $k_{T}$ in this kinematic configuration, is kept. The variable $l^{+}$in $K$ is then integrated up to infinity, such that the scale $(1-x) p^{+}$disappears.

Equation (91) is Fourier transformed into the impact parameter $b$ space, with the definition $\int \Phi\left(x, k_{T}\right) \exp \left(i \mathbf{k}_{T}\right.$. b) $d^{2} k_{T} /(2 \pi)^{2} \equiv \Phi(x, b)$. The convolution in the transverse-momentum space in Eq. (11) then becomes a product under the Fourier transformation. In the intermediate $x$ region $\Phi$ involves two scales, the large $x p^{+}$ 
that characterizes the hard kernel $G$ and the small $1 / b$ that characterizes the soft kernel $K$. The master equation (9) becomes

$$
p^{+} \frac{d}{d p^{+}} \Phi(x, b)=2\left[K\left(1 /(b \mu), \alpha_{s}(\mu)\right)+G\left(x p^{+} \nu / \mu, \alpha_{s}(\mu)\right)\right] \Phi(x, b),
$$

whose solution with $\nu=1$ leads to the $k_{T}$ resummation

$$
\Phi(x, b)=\Delta_{k}(x, b) \Phi_{i}(x)
$$

with the Sudakov exponential

$$
\Delta_{k}(x, b)=\exp \left[-2 \int_{1 / b}^{x p^{+}} \frac{d p}{p} \int_{1 / b}^{p} \frac{d \mu}{\mu} \gamma_{K}\left(\alpha_{s}(\mu)\right)\right],
$$

and the initial condition $\Phi_{i}$ of the Sudakov evolution. The anomalous dimension of $K, \lambda_{K}=\mu d \delta K / d \mu$, is given, up to two loops, by [27]

$$
\gamma_{K}=\frac{\alpha_{s}}{\pi} C_{F}+\left(\frac{\alpha_{s}}{\pi}\right)^{2} C_{F}\left[C_{A}\left(\frac{67}{36}-\frac{\pi^{2}}{12}\right)-\frac{5}{18} n_{f}\right],
$$

with $n_{f}$ being the number of quark flavors and $C_{A}=3$ being a color factor.

The $k_{T}$ resummation effect on the low $p_{T}$ spectra of the direct photon production has been analyzed [28]. The initial-state and final-state radiations are constrained in the low $p_{T}$ region, where the $k_{T}$ resummation is necessary for improving the perturbation theory. Figure 4 shows the deviation, (Data -Theory)/Theory, of the next-to-leading-order (NLO) pQCD predictions, obtained using the CTEQ4M PDFs [29], from the experimental data as a function of $x_{t}=2 p_{T} / \sqrt{s}, \sqrt{s}$ being the center-of-mass energy. The deviation is huge as expected, especially at low $x_{t}$ of each set of the data. After including the $k_{T}$ resummation effect [28], it is clear that a significant improvement on the agreement between the theoretical predictions and the data is achieved. As to the intermediate- and high- $p_{T}$ regions of the direct photon production, NLO pQCD works reasonably well in accommodating the data as shown in [30]. The threshold resummation effect, which will be introduced in the next subsection, is more relevant in these regions: it slightly improves the consistency between predictions and the data [30].

In the small $x$ region with $x p^{+} \sim k_{T}$, or $x p^{+} \sim 1 / b$ in the $b$ space, the two-scale case reduces to the singlescale one. In this region contributions from gluonic partons dominate, so $\Phi$ represents the gluon TMD below. The source of double logarithms, i.e., the integral containing the anomalous dimension $\gamma_{K}$, is less important. Because only the soft scale exists, one drops the hard kernel $G$, and keeps the soft kernel with an ultraviolet cutoff. The right-hand side of Eq. (9) becomes

$$
\begin{aligned}
\bar{\Phi}\left(x, k_{T}\right)= & -i g^{2} N_{c} \int \frac{d^{4} l}{(2 \pi)^{4}} \frac{\hat{n} \cdot v}{n \cdot l v \cdot l}\left[\frac{\Theta\left(k_{T}^{2}-l_{T}^{2}\right)}{l^{2}} \Phi\left(x, k_{T}\right)\right. \\
& \left.+2 \pi i \delta\left(l^{2}\right) \phi\left(x,\left|\mathbf{k}_{T}+\mathbf{l}_{T}\right|\right)\right],
\end{aligned}
$$

where the color factor $C_{F}$ has been replaced by $N_{c}$ for the gluon TMD. The step function $\Theta$ introduces the ultraviolet cutoff on $l_{T}$ mentioned above. To make variation in $x$ via variation in $p^{+}$, a fixed parton momentum is assumed. Under this assumption, the momentum fraction $x$ is proportional to $1 / p^{+}$, and one has $p^{+} d \Phi / d p^{+}=-x d \Phi / d x \Phi$ [31]. Performing the integrations over $l^{+}$and $l^{-}$in Eq. (17), the master equation (9) reduces to the BFKL equation [32],

$$
\frac{d \phi\left(x, k_{T}\right)}{d \ln (1 / x)}=\bar{\alpha}_{s} \int \frac{d^{2} l_{T}}{\pi l_{T}^{2}}\left[\phi\left(x,\left|\mathbf{k}_{T}+\mathbf{l}_{T}\right|\right)-\Theta\left(k_{T}^{2}-l_{T}^{2}\right) \phi\left(x, k_{T}\right)\right],
$$

with the coupling constant $\bar{\alpha}_{s}=N_{c} \alpha_{s} / \pi$.

A remarkable prediction of the above LO BFKL equation is that a high-energy cross section increases with the center-of-mass energy,

$$
\sigma \approx \frac{1}{t}\left(\frac{s}{t}\right)^{\omega_{P}-1}
$$



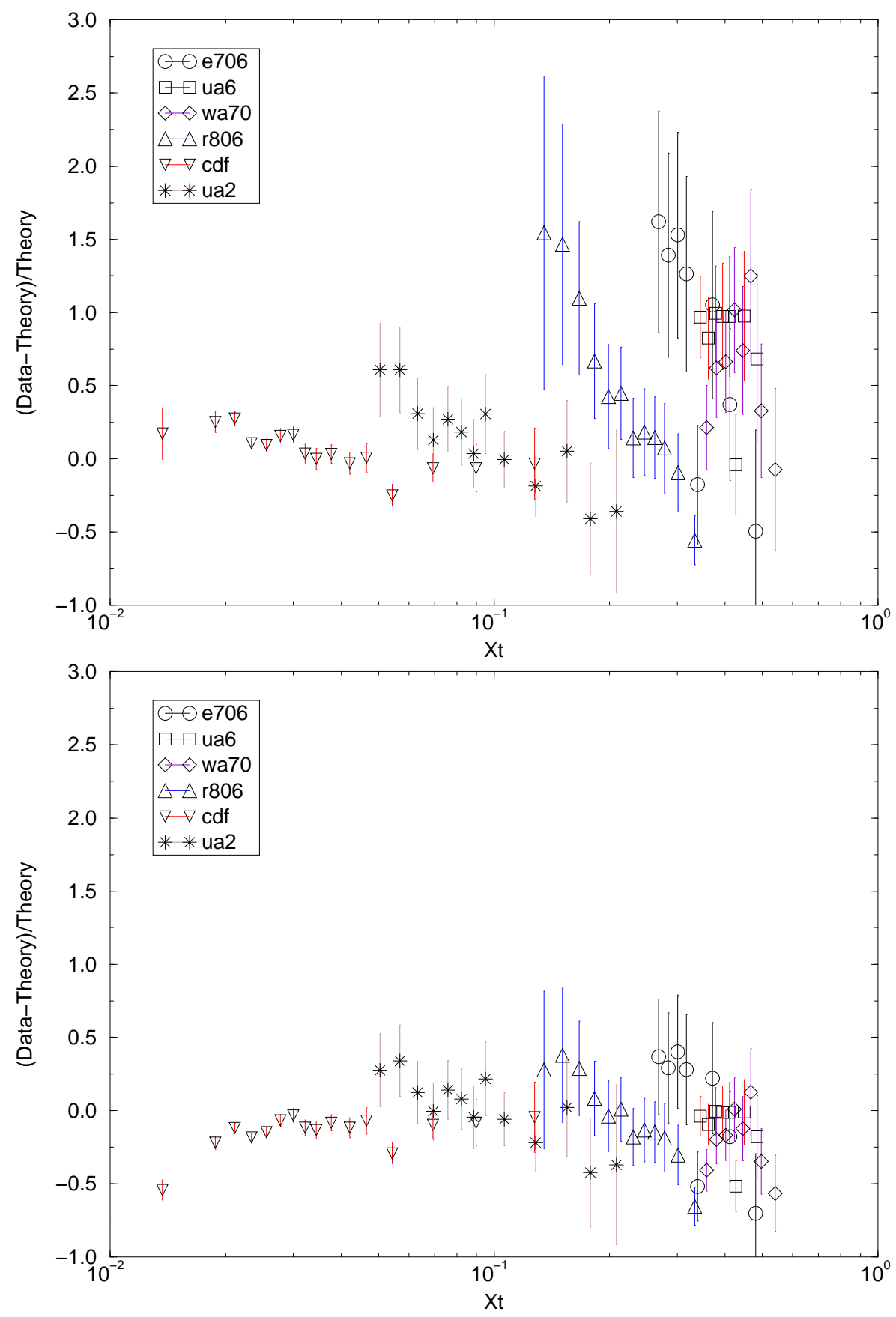

FIG. 4: Low $p_{T}$ direct photon spectra before (upper) and after (lower) including the $k_{T}$ resummation effect.

with the momentum transfer squared $t$. It turns out that Eq. (19), with the Pomeron intercept $\omega_{P}-1=4 \bar{\alpha}_{s} \ln 2$, violates the Froissart (unitarity) bound $\sigma<$ const. $\times \ln ^{2}$ 33]. The unsatisfactory prediction of the LO BFKL equation called for the NLO corrections [34], which were, however, found to be dramatic [35]: the NLO effect is nearly as large as the LO result for $x \sim 0.001$, and becomes dominant at lower $x$. It even turns the derivative of the structure function $d F_{L} / d \ln Q^{2}$ negative below $x \sim 0.0001$. That is, the perturbative solution is not at all stable. 


\section{THRESHOLD RESUMMATION AND DGLAP EQUATION}

We then consider the soft approximation corresponding to the $k_{T}$ ordering of real gluon emissions in a ladder diagram. Assume that a parton without the transverse momentum, carries $-\mathbf{l}_{1 T}$ after emitting a gluon of

longitudinal momentum $l_{1}^{+}$and transverse momentum $\mathbf{l}_{1 T}$, and then carries $-\mathbf{l}_{1 T}-\mathbf{l}_{2 T}$ after emitting a gluon of longitudinal momentum $l_{2}^{+}$and transverse momentum $\mathbf{l}_{2 T}$. In the kinematic configuration with $l_{2 T} \gg l_{1 T}$ and $l_{2}^{+} \sim l_{1}^{+}$, the final parton momentum can be approximated by $-\mathbf{l}_{2 T}-\mathbf{l}_{1 T} \approx-\mathbf{l}_{2 T}$, such that the loop integral associated with the first gluon emission involves only $l_{1 T}$, and can be worked out straightforwardly, giving a logarithm. The loop integral associated with the second gluon emission involves only $l_{2 T}$, and also gives a logarithm. Therefore, a ladder diagram with $N$ rung gluons generates the logarithmic correction $\left(\alpha_{s} L\right)^{N}$ under the above $k_{T}$ ordering. In this case $\Phi$ is independent of $l_{T}$, and we have the approximation for the real gluon emission in Eq. (11)

$$
\Phi\left(x+l^{+} / p^{+},\left|\mathbf{k}_{\mathbf{T}}+\mathbf{l}_{\mathbf{T}}\right|\right) \approx \Phi\left(x+l^{+} / p^{+}, k_{T}\right),
$$

in which $x$ and $l^{+} / p^{+}$are of the same order. The dependence on $k_{T}$ can then be integrated out from both sides of the master equation (9), and the TMD $\Phi$ reduces to the PDF $\phi$. Similarly, the soft contribution $\bar{\Phi}_{s}$ in Eq. (10) reduces to $\bar{\phi}_{s}$. The scale $k_{T}$ disappears, and the scale $(1-x) p^{+}$is retained.

The Mellin transformation is employed to bring $\bar{\phi}_{s}$ from the momentum fraction $x$ space to the moment $N$ space,

$$
\bar{\phi}_{s}(N)=\int_{0}^{1} d x x^{N-1} \bar{\phi}_{s}(x)
$$

under which the $l^{+}$integration decouples. In the large $x$ region $\phi$ involves two scales, the large $x p^{+} \sim p^{+}$from the hard kernel $G$ and the small $(1-x) p^{+} \sim p^{+} / N$ from the soft kernel $K$. To sum $\ln (1 / N)$, we rewrite the derivative $p^{+} d \phi / d p^{+}$as

$$
p^{+} \frac{d \phi}{d p^{+}}=\frac{p^{+}}{N} \frac{d \phi}{d\left(p^{+} / N\right)}
$$

The solution of the master equation (9) then gives the threshold resummation,

$$
\phi(N)=\Delta_{t}(N) \phi_{i}
$$

with the exponential

$$
\Delta_{t}(N)=\exp \left[-2 \int_{p^{+} / N}^{p^{+}} \frac{d p}{p} \int_{p^{+}}^{p} \frac{d \mu}{\mu} \gamma_{K}\left(\alpha_{s}(\mu)\right)\right]
$$

or its equivalent expression

$$
\Delta_{t}(N)=\exp \left[\int_{0}^{1} d z \frac{1-z^{N-1}}{1-z} \int_{(1-z)^{2}}^{1} \frac{d \lambda}{\lambda} \gamma_{K}\left(\alpha_{s}\left(\sqrt{\lambda} p^{+}\right)\right)\right]
$$

It has been investigated that Eq. (24) becomes reliable as $N$ is about or greater than $O\left(10^{2}\right)$ at the Tevatron energy [36]. Equation (24) is accurate up to next-to-leading logarithms (NLL), so corrections to it appear at next-to-next-to-leading logarithms (NNLL) and at powers of $1 / N$.

An application of the threshold resummation is found in the analysis of the top-quark pair production, which was performed at the NNLL accuracy [37]. It has been observed that the threshold resummation effect enhances the NLO total cross section by few percents, which make an impact on the determination of the top quark mass.

In the intermediate $x$ region the two-scale case reduces to the single-scale one because of $x p^{+} \sim(1-x) p^{+}$, and the source of double logarithms is less important. Without the Mellin transformation, the sum in Eq. (10), with the approximation in Eq. (20) being inserted, leads to the DGLAP equation [31],

$$
p^{+} \frac{d}{d p^{+}} \phi(x)=\int_{x}^{1} \frac{d \xi}{\xi} P(x / \xi) \phi(\xi),
$$



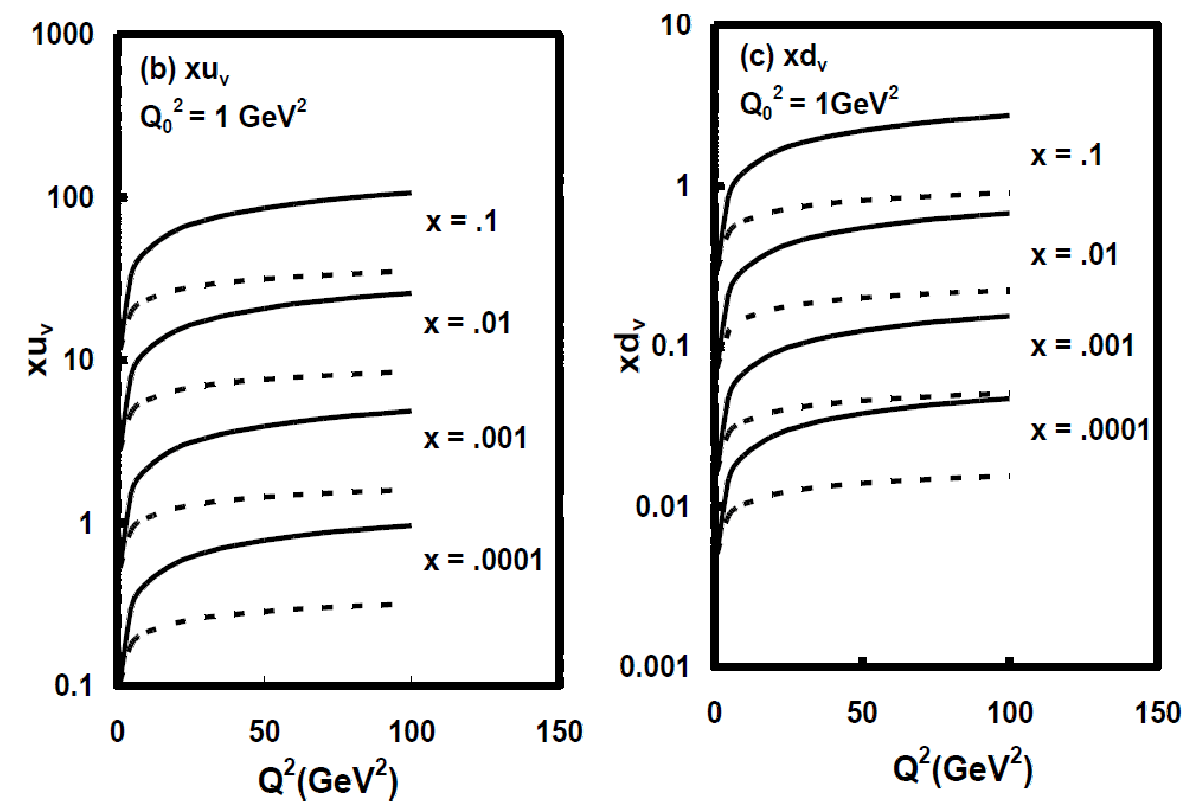

FIG. 5: $Q^{2}$ evolutions of the valence quark PDFs for some parameter values in the DGLAP solutions (solid and dashed lines).

with the kernel

$$
P(z)=\frac{\alpha_{s}\left(p^{+}\right)}{\pi} C_{F} \frac{2}{(1-z)_{+}}
$$

where the variable change $\xi=x+l^{+} / p^{+}$has been made. The argument of $\alpha_{s}$, i.e, the factorization scale $\mu$, has been set to the scale $x p^{+} \sim(1-x) p^{+} \sim O\left(p^{+}\right)$. Note that the kernel $P$ differs from the splitting function $P_{q q}$

$$
P_{q q}^{(1)}(x)=C_{F}\left(\frac{1+x^{2}}{1-x}\right)_{+}
$$

by the term $\left(z^{2}-1\right) /(1-z)_{+}$, which is finite in the $z \rightarrow 1$ limit. The reason is that the real gluon emission was evaluated under the soft approximation as deriving $P$, while it was calculated exactly as deriving $P_{q q}$.

Gluon emissions cause the mixing between the quark and gluon PDFs, giving the complete set of DGLAP equations with four splitting functions

$$
\frac{\partial}{\partial \ln Q^{2}}\left(\begin{array}{c}
\phi_{q} \\
\phi_{g}
\end{array}\right)=\left(\begin{array}{cc}
P_{q q} & P_{q g} \\
P_{g q} & P_{g g}
\end{array}\right) \otimes\left(\begin{array}{c}
\phi_{q} \\
\phi_{g}
\end{array}\right) .
$$

The evolution of the $u$-quark and $d$-quark PDFs in $Q^{2}$ predicted by the LO DGLAP equation [38] is shown in Fig. 5], where the inputs at the initial scale $Q_{0}=1 \mathrm{GeV}$ were taken from MRST2001 [39]. It is observed that the valence quark PDFs increase with $Q^{2}$ at small $x$, namely, they become broader with $Q^{2}$.

\section{JOINT RESUMMATION AND CCFM EQUATION}

At last, a unified resummation formalism for large and intermediate $x$ and a unified evolution equation for intermediate and small $x$ can be derived by retaining the $l^{+}$and $l_{T}$ dependencies of $\Phi$ in Eq. (11), which corresponds to the so-called angular ordering. In this case both the Fourier and Mellin transformations are applied to Eq. (11), leading to

$$
\bar{\Phi}_{s}(N, b)=K\left(p^{+} /(N \mu), 1 /(b \mu), \alpha_{s}(\mu)\right) \Phi(N, b),
$$


with the soft kernel [6]

$$
\begin{aligned}
K= & -i g^{2} C_{F} \mu^{\epsilon} \int_{0}^{1} d z \int \frac{d^{4-\epsilon} l}{(2 \pi)^{4-\epsilon}} \frac{\hat{n} \cdot v}{n \cdot l v \cdot l}\left[\frac{\delta(1-z)}{l^{2}}\right. \\
& \left.+2 \pi i \delta\left(l^{2}\right) \delta\left(1-z-\frac{l^{+}}{p^{+}}\right) z^{N-1} e^{i \mathbf{l}_{T} \cdot \mathbf{b}}\right]-\delta K, \\
= & \frac{\alpha_{s}(\mu)}{\pi} C_{F}\left[\ln \frac{1}{b \mu}-K_{0}\left(\frac{2 \nu p^{+} b}{N}\right)\right],
\end{aligned}
$$

$K_{0}$ being the modified Bessel function. As $p^{+} b \gg N$, we have $K_{0} \rightarrow 0$, and the soft scale inferred by the above expression approaches $1 / b$ for the $k_{T}$ resummation. As $N \gg p^{+} b$, we have $K_{0} \approx-\ln \left(\nu p^{+} b / N\right)$, and the soft scale approaches $p^{+} / N$ for the threshold resummation.

Following the procedures similar to Eqs. (13)-(15), we derive the joint resummation

$$
\Phi(N, b)=\Delta_{u}(N, b) \Phi_{i},
$$

with the exponential

$$
\Delta_{u}(N, b)=\exp \left[-2 \int_{p^{+} \chi^{-1}(N, b)}^{p^{+}} \frac{d p}{p} \int_{p^{+} \chi^{-1}(1, b)}^{p} \frac{d \mu}{\mu} \gamma_{K}\left(\alpha_{s}(\mu)\right)\right] .
$$

The dimensionless function [7]

$$
\chi(N, b)=\left(N+\frac{p^{+} b}{2}\right) e^{\gamma_{E}},
$$

is motivated by the limits discussed above. It is apparent that Eq. (33) reduces to Eq. (15) and Eq. (24) in the $b \rightarrow \infty$ and $N \rightarrow \infty$ limits, respectively. The effect from the joint resummation on the $q_{T}$ spectra of selectron pairs produced at the LHC with $\sqrt{S}=14 \mathrm{TeV}$ has been investigated in [40]. It is seen in Fig. [6 that the joint and $k_{T}$ resumations exhibit a similar behavior in the small- $q_{T}$ region as expected, but the jointly-resummed cross section is about 5\%-10\% lower than the $k_{T}$-resummed cross section in the range $50 \mathrm{GeV}<q_{T}<100 \mathrm{GeV}$.

In the intermediate and small $x$ regions, it is not necessary to resum the double logarithms $\ln ^{2}(1 / N)$. After extracting the $k_{T}$ resummation, the remaining single-logarithmic summation corresponds to a unification of the DGLAP and BFKL equations, since both the $l^{+}$and $l_{T}$ dependencies have been retained. The function $\Phi\left(x+l^{+} / p^{+}, b\right)$ in Eq. (11) is reexpressed, after the Fourier transformation, as

$$
\begin{aligned}
\Phi\left(x+l^{+} / p^{+}, b\right) & =\Theta\left((1-x) p^{+}-l^{+}\right) \Phi(x, b) \\
+ & {\left[\Phi\left(x+l^{+} / p^{+}, b\right)-\Theta\left((1-x) p^{+}-l^{+}\right) \Phi(x, b)\right] . }
\end{aligned}
$$

The contribution from the first term is combined with the first term in Eq. (11), giving the soft kernel $K$ for the $k_{T}$ resummation. The second term in Eq. (35) contributes

$$
-i N_{c} g^{2} \int \frac{d^{4} l}{(2 \pi)^{4}} \frac{\hat{n} \cdot v}{n \cdot l v \cdot l} 2 \pi i \delta\left(l^{2}\right) e^{i \mathbf{l}_{T} \cdot \mathbf{b}}\left[\Phi\left(x+l^{+} / p^{+}, b\right)-\Theta\left((1-x) p^{+}-l^{+}\right) \Phi(x, b)\right],
$$

which will generate the splitting function below. The color factor has been replaced by $N_{c}$, since the gluon TMD is considered here.

The master equation (9) then becomes

$$
\begin{aligned}
p^{+} \frac{d}{d p^{+}} \Phi(x, b)= & -2\left[\int_{1 / b}^{x p^{+}} \frac{d \mu}{\mu} \gamma_{K}\left(\alpha_{s}(\mu)\right)-\bar{\alpha}_{s}\left(x p^{+}\right) \ln \left(p^{+} b\right)\right] \Phi(x, b) \\
& +2 \bar{\alpha}_{s}\left(x p^{+}\right) \int_{x}^{1} d z P_{g g}(z) \Phi(x / z, b),
\end{aligned}
$$




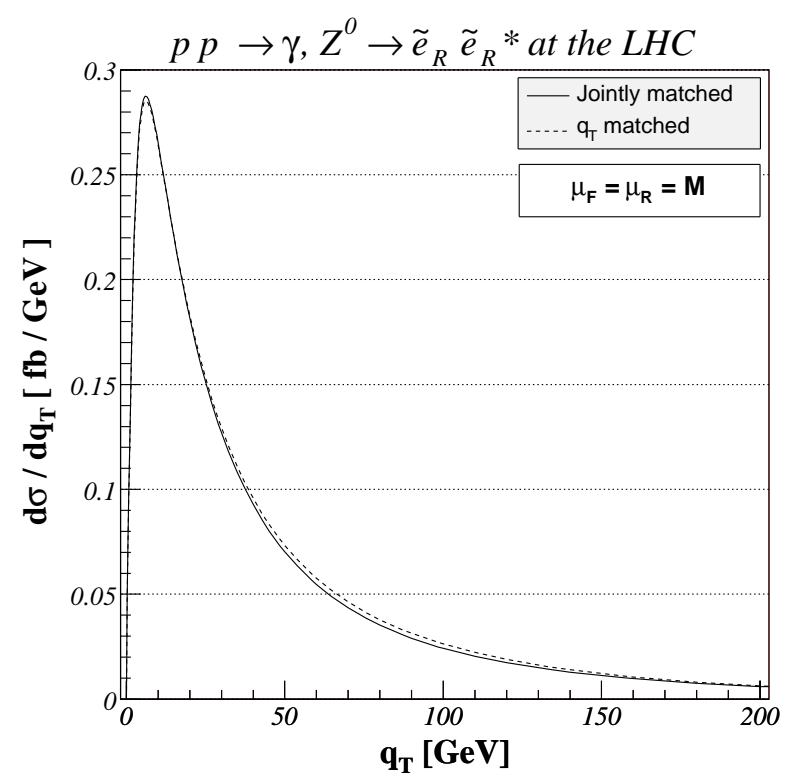

FIG. 6: Transverse-momentum distribution of selectron pairs at the LHC in the framework of joint (full) and $k_{T}$ (dotted) resummations.

with the splitting function

$$
P_{g g}=\left[\frac{1}{(1-z)_{+}}+\frac{1}{z}-2+z(1-z)\right]
$$

obtained from Eq. (36). The term $-2+z(1-z)$ finite as $z \rightarrow 0$ and $z \rightarrow 1$ has been added. The exponential $\Delta$ is extracted from the $k_{T}$ resummation,

$$
\Delta\left(x, b, Q_{0}\right)=\exp \left(-2 \int_{x Q_{0}}^{x p^{+}} \frac{d p}{p}\left[\int_{1 / b}^{p} \frac{d \mu}{\mu} \gamma_{K}\left(\alpha_{s}(\mu)\right)-\bar{\alpha}_{s}(p) \ln \frac{p b}{x}\right]\right),
$$

$Q_{0}$ being an arbitrary low energy scale. It is trivial to justify by substitution that the solution is given by

$$
\begin{aligned}
\Phi(x, b)= & \Delta\left(x, b, Q_{0}\right) \Phi_{i} \\
& +2 \int_{x}^{1} d z \int_{Q_{0}}^{p^{+}} \frac{d \mu}{\mu} \bar{\alpha}_{s}(x \mu) \Delta_{k}(x, b) P_{g g}(z) \Phi(x / z, b),
\end{aligned}
$$

which can be regarded as a modified version of the CCFM equation [10].

\section{JET MASS DISTRIBUTION}

Jets, abundantly produced at colliders [41], carry information of hard scattering and parent particles, which is crucial for particle identification and new physics search. Study of jet physics usually relies on event generators, which, however, suffer ambiguity from parameter tuning. Hence, we are motivated to establish an alternative approach free of the ambiguity. I will demonstrate that jet dynamics can be explored and jet properties can be predicted in the resummation formalism with the Wilson lines off the light cone.

We start from the dijet production in the $e^{-} e^{+}$annihilation, which is part of its total cross section. The physical dijet final state contains two jet cones of half angle $\delta$ and isotropic soft gluons within the energy resolution $\epsilon Q, Q$ being the $e^{-} e^{+}$invariant mass. With the constrained phase space for real gluons, the infrared cancellation is not complete, and logarithmic enhancement appears. The explicit NLO calculations imply that 
the isotropic soft gluons give a contribution proportional to $2 \ln ^{2}(2 \epsilon Q / \mu)-\pi^{2} / 6$, the collinear gluons in the cones with energy higher than the resolution give $-3 \ln (Q \delta / \mu)-2 \ln ^{2}(2 \epsilon)-4 \ln (Q \delta / \mu) \ln (2 \epsilon)+17 / 4-\pi^{2} / 3$, and the virtual corrections contribute $-2 \ln ^{2}(Q / \mu)+3 \ln (Q / \mu)-7 / 4+\pi^{2} / 6$. The total NLO corrections indicate that the dijet cross section is infrared finite, but logarithmically enhanced [42]:

$$
3 \ln \delta+4 \ln \delta \ln (2 \epsilon)+\frac{\pi^{2}}{3}-\frac{5}{2},
$$

where the double logarithm $\ln \delta \ln (2 \epsilon)$ is attributed to the overlap of the collinear and soft logarithms.

We then explain the factorization of a jet from DIS, whose production is expected to be enhanced by collinear dynamics as indicated by Eq. (41). A jet is formed, as the gluon emitted by the initial-state or final-state quark is collimated to the final-state quark. The restricted phase space of the final-state quark and the gluon in a small angular separation renders an incomplete cancellation between the virtual and real corrections. In this kinematic configuration the initial-state quark propagator can be eikonalized, such that collinear gluons are detached from the initial-state quark and absorbed into a jet function. To all orders, the collinear gluons are collected by the Wilson link with an arbitrary vector $n$. The collinear gluon emitted by the final-state quark can be factorized into the jet function straightforwardly by applying the Fierz transformation. A more sophisticated factorization formula for the jet production in the DIS is then written as a convolution of a hard kernel $H$ with a PDF and a jet function $J$. $H$ denotes the contribution with the collinear pieces for the initial and final states being subtracted.

The light-quark and gluon jet functions are defined by [43]

$$
\begin{aligned}
J_{q}\left(M_{J}^{2}, P_{T}, \nu^{2}, R, \mu^{2}\right)= & \frac{(2 \pi)^{3}}{2 \sqrt{2}\left(P_{J}^{0}\right)^{2} N_{c}} \sum_{N_{J}} \operatorname{Tr}\left\{\xi\left\langle 0\left|q(0) W^{(\bar{q}) \dagger}\right| N_{J}\right\rangle\left\langle N_{J}\left|W^{(\bar{q})} \bar{q}(0)\right| 0\right\rangle\right\} \\
& \times \delta\left(M_{J}^{2}-\hat{M}_{J}^{2}\left(N_{J}, R\right)\right) \delta^{(2)}\left(\hat{e}-\hat{e}\left(N_{J}\right)\right) \delta\left(P_{J}^{0}-\omega\left(N_{J}\right)\right), \\
J_{g}\left(M_{J}^{2}, P_{T}, \nu^{2}, R, \mu^{2}\right)= & \frac{(2 \pi)^{3}}{2\left(P_{J}^{0}\right)^{3} N_{c}} \sum_{N_{J}}\left\langle 0\left|\xi_{\sigma} F^{\sigma \nu}(0) W^{(g) \dagger}\right| N_{J}\right\rangle\left\langle N_{J}\left|W^{(g)} F_{\nu}^{\rho}(0) \xi_{\rho}\right| 0\right\rangle \\
& \times \delta\left(M_{J}^{2}-\hat{M}_{J}^{2}\left(N_{J}, R\right)\right) \delta^{(2)}\left(\hat{e}-\hat{e}\left(N_{J}\right)\right) \delta\left(P_{J}^{0}-\omega\left(N_{J}\right)\right),
\end{aligned}
$$

where $\left|N_{J}\right\rangle$ denotes the final state with $N_{J}$ particles within the cone of size $R$ centered in the direction of the unit vector $\hat{e}, \hat{M}_{J}\left(N_{J}, R\right)\left(\omega\left(N_{J}\right)\right)$ is the invariant mass (total energy) of all $N_{J}$ particles, and $\mu$ is the factorization scale. The above jet functions absorb the collinear divergences from all-order radiations associated with the energetic light jet of momentum $P_{J}^{\mu}=P_{J}^{0} v^{\mu}$, in which $P_{J}^{0}$ is the jet energy, and the vector $v$ is given by $v^{\mu}=(1, \beta, 0,0)$ with $\beta=\sqrt{1-\left(M_{J} / P_{J}^{0}\right)^{2}} . \xi^{\mu}=(1,-1,0,0)$ is a vector on the light cone. The coefficients in Eq. (42) have been chosen, such that the LO jet functions are equal to $\delta\left(M_{J}^{2}\right)$ in a perturbative expansion.

Underlying events include everything but hard scattering, such as initial-state radiation, final-state radiation, and multiple parton interaction (MPI). The Wilson lines in Eq. (42) have collected gluons radiated from both initial states and other final states in a scattering process, and collimated to the light-particle jets. Gluon exchanges between the quark fields $q$ (or the gluon fields $F^{\sigma \nu}$ and $F_{\nu}^{\rho}$ ) correspond to the final-state radiations. Both the initial-state and final-state radiations are leading-power effects in the factorization theorem, and have been included in the jet function definition. A chance of involving more partons in hard scattering is low, so the contribution from MPI is regarded as being subleading-power. This contribution should be excluded from data, but it is certainly difficult to achieve in experiments. Nevertheless, it still makes sense to compare predictions for jet observables based on Eq. (42) at the current leading-power accuracy with experimental data. At last, pile-up events must be removed in experiments [44], since they cannot be handled theoretically so far.

The NLO diagrams for the light-quark and gluon jet functions are displayed in Figs. 7 and 8 , respectively. Evaluating the jet functions up to NLO, a divergence, compared to PYTHIA predictions, is observed at small jet invariant mass $M_{J}$ as shown in Fig. 9, that implies the nonperturbtive nature of the jet functions. The total NLO corrections in Mellin space indicate the existence of double logarithms, which demand the implementation of the resummation technique. Both the angular and energy resolutions are related to the jet mass: when $M_{J}$ is not zero, particles in a jet cannot be completely collimated, and the jet must have finite minimal energy. This accounts for the source of the double logarithms. Recall that low $p_{T}$ spectra of direct photons, dominated by soft and collinear radiations, are treated by the $k_{T}$ resummation. The jet invariant mass is attributed to soft and collinear radiations, so the mass distribution can also be derived in the resummation formalism. 


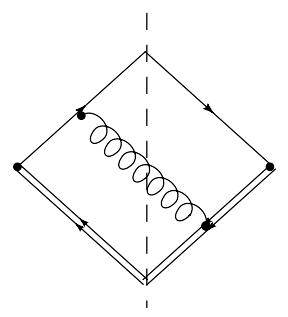

(a)

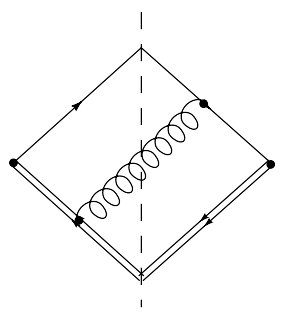

(b)

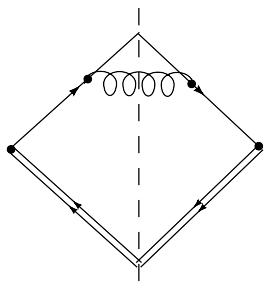

(c)

FIG. 7: Some NLO real corrections to the quark jet function.

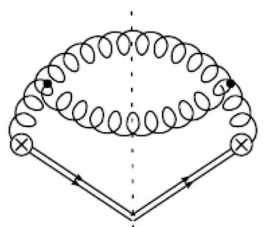

(a)

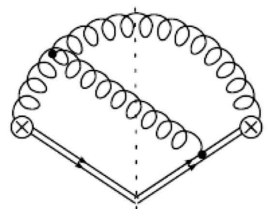

(d)

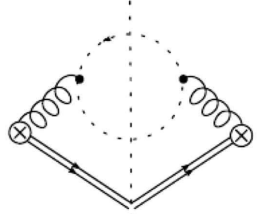

(b)

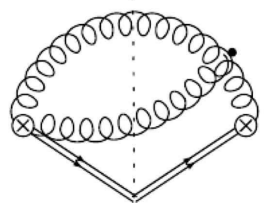

(e)

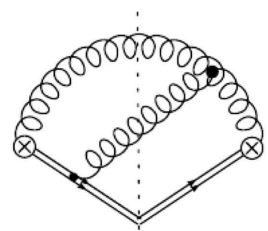

(c)

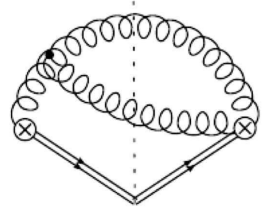

(f)

FIG. 8: Some NLO real corrections to the gluon jet function, where the dashed line represents a ghost field.

Varying the Wilson line direction $n$, we derive the differential equation for the light-quark jet function [12]

$$
-\frac{n^{2}}{v \cdot n} v_{\alpha} \frac{d}{d n_{\alpha}} J_{q}\left(M_{J}^{2}, P_{T}, \nu^{2}, R, \mu^{2}\right)=2(K+G) \otimes J_{q}\left(M_{J}^{2}, P_{T}, \nu^{2}, R, \mu^{2}\right) .
$$

The above equation implies that the soft gluons in $K$ are associated with the jet function $J$, a prescription consistent with the anti- $k_{T}$ algorithm. The strategy is to evolve $n$, i.e., $\nu^{2}$ from a low value $\nu_{\text {in }}^{2} \sim O(1 / N)$ to a large value $\nu_{\mathrm{fi}}^{2} \sim O(1)$. The former defines the initial condition of the jet function, which can be evaluated up to a fixed order, because of the vanishing of the $\operatorname{logarithm} \ln \left(\nu^{2} N\right)$. The latter reproduces all important logarithms in the jet function, such that the solution of Eq. (43) collects their resummation. One then convolutes the light-quark and gluon jet functions with the constituent cross sections of LO partonic dijet processes at the Tevatron and the PDF CTEQ6L [45]. The resummation predictions for the jet mass distributions at $R=0.4$ and $R=0.7$ are compared to the Tevatron CDF data 13] in Fig. 10 12] with the kinematic cuts $P_{T}>400 \mathrm{GeV}$ and the rapidity interval $0.1<|Y|<0.7$. The abbreviation NLL refers to the accuracy of the resummation at next-to-leading logarithm, and NLO to the accuracy of the initial condition of the jet function solved from Eq. (43). The consistency of the resummation results with the CDF data is satisfactory.

\section{JET ENERGY PROFILE}

It is known that a top quark produced almost at rest at the Tevatron can be identified by measuring isolated jets from its decay. However, this strategy does not work for identifying a highly-boosted top quark produced at the LHC. It has been observed that an ordinary high-energy QCD jet [46, 47] can have an invariant mass close to the top quark mass. A highly-boosted top quark, producing only a single jet, is then difficult to be distinguished from a QCD jet. This difficulty also appears in the identification of a highly-boosted new-physics resonance decaying into standard-model particles, or Higgs boson decaying into a bottom-quark pair. Hence, additional 


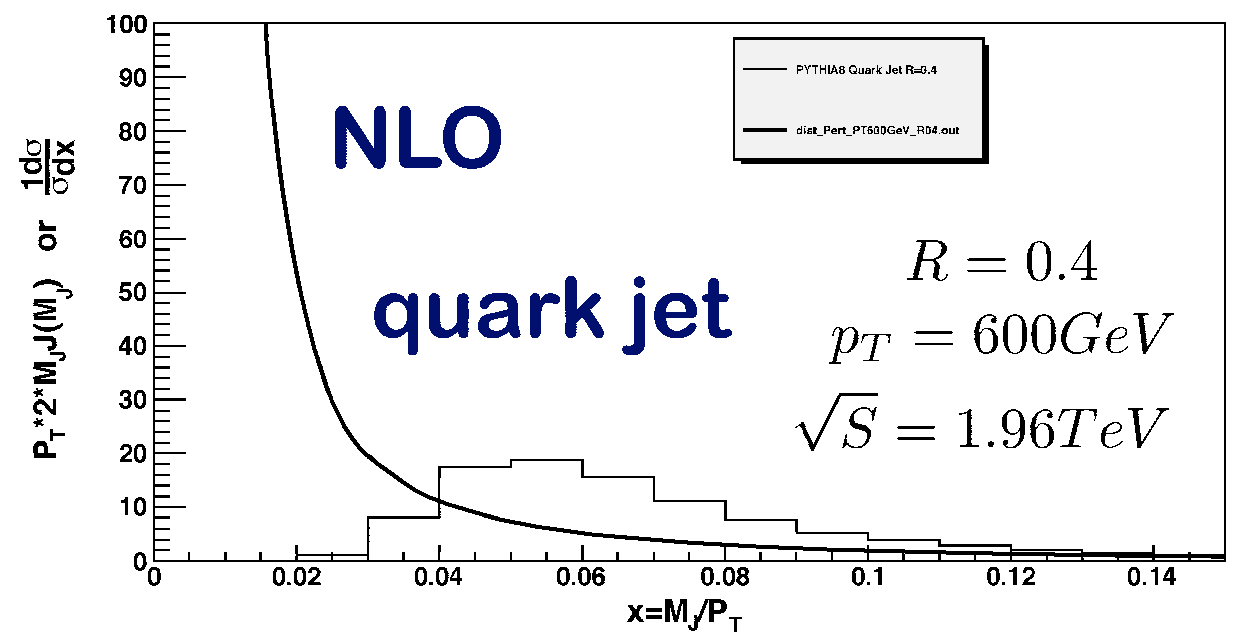

FIG. 9: Jet mass distribution at NLO.

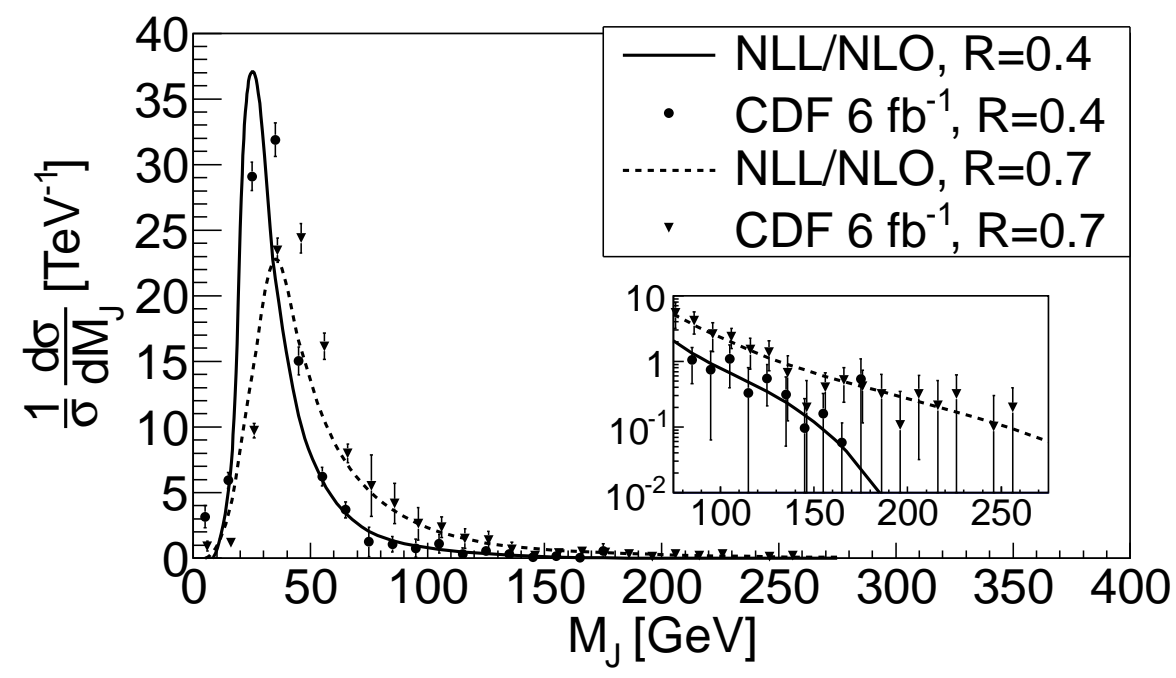

FIG. 10: Comparison of resummation predictions for the jet mass distributions to Tevatron CDF data with the kinematic cuts $P_{T}>400 \mathrm{GeV}$ and $0.1<|Y|<0.7$ at $R=0.4$ and $R=0.7$. The inset shows the detailed comparison in large jet mass region.

information needs to be extracted from jet internal structures in order to improve the jet identification at the LHC. The quantity, called planar flow [48], has been proposed for this purpose, which utilizes the geometrical shape of a jet: a QCD jet with large invariant mass mainly involves one-to-two splitting, so it leaves a linear energy deposition in a detector. A top-quark jet, proceeding with a weak decay, mainly involves one-to-three splitting, so it leaves a planar energy deposition. Measuring this additional information, it has been shown with event generators that the top-quark identification can be improved to some extent. Investigations on various observables associated with jet substructures are usually done using event generators. For a review on recent theoretical progress and the latest experimental results in jet substructures, see [49].

Here I focus on a jet substructure, called the energy profile, and explain how to calculate it in the resummation formalism [12]. This quantity describes the energy fraction accumulated in the cone of size $r$ within a jet cone $R$, i.e., $r<R$. Its explicit definition is given by [14]

$$
\Psi(r)=\frac{1}{N_{J}} \sum_{J} \frac{\sum_{r_{i}<r, i \in J} P_{T i}}{\sum_{r_{i}<R, i \in J} P_{T i}},
$$


with the normalization $\Psi(R)=1$, where $P_{T i}$ is the transverse momentum carried by particle $i$ in the jet $J$, and $r_{i}<r\left(r_{i}<R\right)$ means the flow of particle $i$ into the jet cone $r(R)$. Different types of jets are expected to exhibit different energy profiles. For example, a light-quark jet is narrower than a gluon jet; that is, energy is accumulated faster with $r$ in a light-quark jet than in a gluon jet. A heavy-particle jet certainly has a distinct energy profile, which can be used for its identification. The importance of higher-order corrections and their resummation for studying a jet energy profile have been first emphasized in [50]. Another approach based on the soft-collinear effective theory and its application to jet production at an electron-positron collider can be found in Refs. [51 53].

We first define the jet energy functions $J_{f}^{E}\left(M_{J}^{2}, P_{T}, \nu^{2}, R, r\right)$ with $f=q(g)$ denoting the light-quark (gluon), which describe the energy accumulation within the cone of size $r<R$. The definition is chosen, such that $J_{f}^{E(0)}=P_{T} \delta\left(M_{J}^{2}\right)$ at LO. The Feynman rules for $J_{f}^{E}$ are similar to those for the jet functions $J_{f}$ at each order of $\alpha_{s}$, except that a sum of the step functions $\sum_{i} k_{i}^{0} \Theta\left(r-\theta_{i}\right)$ is inserted, where $k_{i}^{0}\left(\theta_{i}\right)$ is the energy (the angle with respect to the jet axis) of particle $i$. For example, the jet energy functions $J_{f}^{E}$ are expressed, at NLO, as

$$
\begin{aligned}
J_{q}^{E(1)}\left(M_{J}^{2}, P_{T}, \nu^{2}, R, r, \mu^{2}\right)= & \frac{(2 \pi)^{3}}{2 \sqrt{2}\left(P_{J}^{0}\right)^{2} N_{c}} \sum_{\sigma, \lambda} \int \frac{d^{3} p}{(2 \pi)^{3} 2 p^{0}} \frac{d^{3} k}{(2 \pi)^{3} 2 k^{0}} \\
& \times\left[p^{0} \Theta\left(r-\theta_{p}\right)+k^{0} \Theta\left(r-\theta_{k}\right)\right] \\
& \times \operatorname{Tr}\left\{\xi\left\langle 0\left|q(0) W^{(\bar{q}) \dagger}\right| p, \sigma ; k, \lambda\right\rangle\left\langle k, \lambda ; p, \sigma\left|W^{(\bar{q})} \bar{q}(0)\right| 0\right\rangle\right\} \\
& \times \delta\left(M_{J}^{2}-(p+k)^{2}\right) \delta^{(2)}\left(\hat{e}-\hat{e}_{\mathbf{p}+\mathbf{k}}\right) \delta\left(P_{J}^{0}-p^{0}-k^{0}\right), \\
J_{g}^{E(1)}\left(M_{J}^{2}, P_{T}, \nu^{2}, R, r, \mu^{2}\right)= & \frac{(2 \pi)^{3}}{2\left(P_{J}^{0}\right)^{3} N_{c}} \sum_{\sigma, \lambda} \int \frac{d^{3} p}{(2 \pi)^{3} 2 p^{0}} \frac{d^{3} k}{(2 \pi)^{3} 2 k^{0}} \\
& \times\left[p^{0} \Theta\left(r-\theta_{p}\right)+k^{0} \Theta\left(r-\theta_{k}\right)\right] \\
& \times\left\langle 0\left|\xi_{\sigma} F^{\sigma \nu}(0) W^{(g) \dagger}\right| p, \sigma ; k, \lambda\right\rangle\left\langle k, \lambda ; p, \sigma\left|W^{(g)} F_{\nu}^{\rho}(0) \xi_{\rho}\right| 0\right\rangle \\
& \times \delta\left(M_{J}^{2}-(p+k)^{2}\right) \delta^{(2)}\left(\hat{e}-\hat{e}_{\mathbf{p}+\mathbf{k}}\right) \delta\left(P_{J}^{0}-p^{0}-k^{0}\right),
\end{aligned}
$$

where the expansion of the Wilson links in $\alpha_{s}$ is understood. The factorization scale is set to $\mu=P_{T}$ to remove the associated logarithms, so its dependence will be suppressed below.

The Mellin-transformed jet energy function $\bar{J}_{q}^{E}$ obeys a similar differential equation [12]

$$
-\frac{n^{2}}{v \cdot n} v_{\alpha} \frac{d}{d n_{\alpha}} \bar{J}_{q}^{E}\left(N=1, P_{T}, \nu^{2}, R, r\right)=2(\bar{K}+G) \bar{J}_{q}^{E}\left(N=1, P_{T}, \nu^{2}, R, r\right),
$$

which can be solved simply. Inserting the solutions to Eq. (46) into Eq. (44), the jet energy profile is derived. Note that a jet energy profile with $N=1$ is not sensitive to the nonperturbative contribution, so the predictions are free of the nonperturbative parameter dependence, in contrast to the case of the jet invariant mass distribution. It has been found that the light-quark jet has a narrower energy profile than the gluon jet, as exhibited in Fig. 11 for $\sqrt{s}=7 \mathrm{TeV}$ and the interval $80 \mathrm{GeV}<P_{T}<100 \mathrm{GeV}$ of the jet transverse momentum. The broader distribution of the gluon jet results from stronger radiations caused by the larger color factor $C_{A}=3$, compared to $C_{F}=4 / 3$ for a light-quark jet.

One then convolutes the light-quark and gluon jet energy functions with the constituent cross sections of the LO partonic subprocess and CTEQ6L PDFs [45] at certain collider energy. The predictions are directly compared with the Tevatron CDF data [14] as shown in Fig. 12. It is evident that the resummation predictions agree well with the data in all $P_{T}$ intervals. The NLO predictions derived from $\bar{J}_{f}^{E(1)}\left(1, P_{T}, \nu_{\mathrm{f}}^{2}, R, r\right)$ are also displayed for comparison, which obviously overshoot the data. The resummation predictions for the jet energy profiles are compared with the LHC CMS data at $7 \mathrm{TeV}$ [15] from the anti- $k_{T}$ jet algorithm [54] in Fig. [13. which are also consistent with the data in various $P_{T}$ intervals. Since one can separate the contributions from the light-quark jet and the gluon jet, the comparison with the CDF and CMS data implies that high-energy (low-energy) jets are mainly composed of the light-quark (gluon) jets. Therefore, a precise measurement of the jet energy profile as a function of jet transverse momentum can be used to experimentally discriminate the production mechanism of jets in association with other particles, such as electroweak gauge bosons, top quarks and Higgs bosons. 


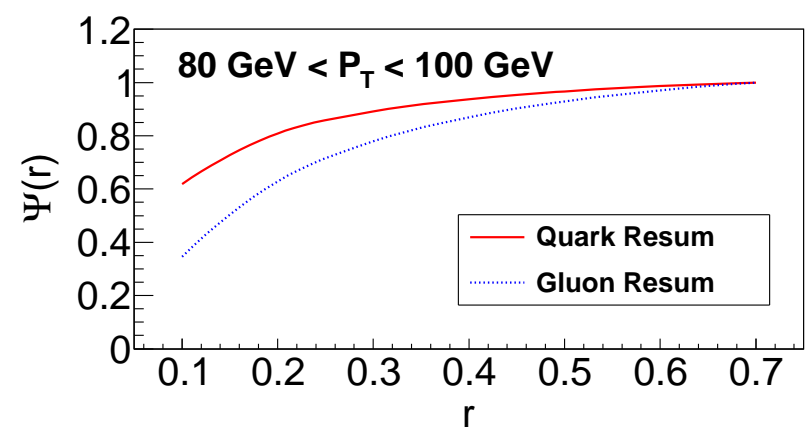

FIG. 11: Resummation predictions for the energy profiles of the light-quark (solid curve) and gluon (dotted curve) jets with $\sqrt{s}=7 \mathrm{TeV}$ and $80 \mathrm{GeV}<P_{T}<100 \mathrm{GeV}$.
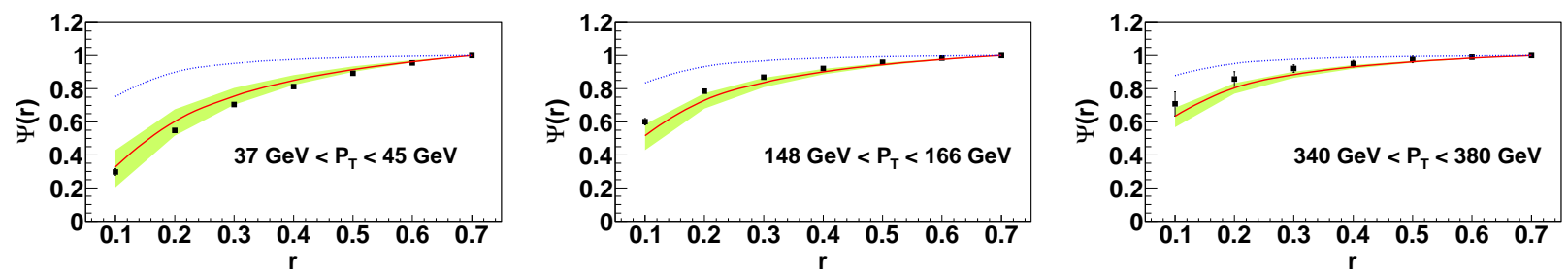

FIG. 12: Comparison of resummation predictions for the jet energy profiles with $R=0.7$ to Tevatron CDF data in various $P_{T}$ intervals. The NLO predictions denoted by the dotted curves are also displayed.
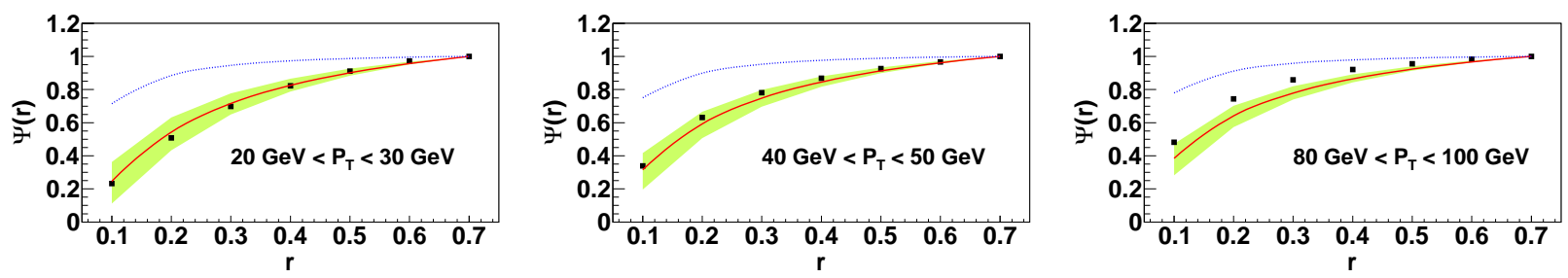

FIG. 13: Resummation predictions for the jet energy profiles with $R=0.7$ compared to LHC CMS data in various $P_{T}$ intervals. The NLO predictions denoted by the dotted curves are also displayed.

\section{SUMMARY}

In this article I have reviewed the resummation through the variation of the Wilson lines off the light cone, and its applications to the derivation of all the known single- and double-logarithm summations, including their unifications. The idea is that the collinear dynamics involved in a collision subprocess is independent of the Wilson line direction $n$, so the variation effect can be factorized out of the subprocess, leading to an evolution equation in $n$. The solution to this evolution equation then resums important infrared logarithms in the subprocess. For the derivations of various logarithmic summations, the point is the treatment of the real gluon contributions to the subdiagram containing the special vertex in the resummation formalism. Simply adopting the soft approximations appropriate in different kinematic regions, i.e., neglecting the $l^{+}$or $l_{T}$ dependence in the TMD associated with the real gluon emission, the formalism reduces to the $k_{T}$ resummation, the BFKL equation, the threshold resummation, or the DGLAP equation. If keeping both the $l^{+}$and $l_{T}$ dependencies, the joint resummation for large $x$ and the CCFM equation for intermediate and small $x$ are obtained. The same technique has been applied to the study of jet substructures, and it has been shown that the invariant mass distributions and the energy profiles of the light-quark and gluon jets can be calculated.

In this framework only the one-loop subdiagrams were evaluated for demonstration, which corresponds to the summation of ladder graphs, or to the summation of real gluon emissions under strong kinematic orderings. To improve the accuracy of resummation, non-ladder graphs and contributions from the configuration without 
strong kinematic orderings need to be included. By computing the subdiagrams to two loops, the former give next-to-leading-logarithmic corrections. The contribution from the region without, for example, the $k_{T}$ ordering is taken into account by keeping the $l_{T}$ dependence of the TMD, similar to the derivation of the BFKL equation appropriate for the multi-Regge region. That is, theoretical extensions of the resummation formalism with the Wilson lines off the light cone are also promising.

\section{Acknowledgments}

This work was supported by the National Science Council of R.O.C. under Grant No. NSC-101-2112-M-001006-MY3 and by the National Center for Theoretical Sciences of R.O.C...

[1] J.C. Collins and D.E. Soper, Nucl. Phys. B193, 381 (1981).

[2] J.C. Collins, D.E. Soper, and G. Sterman, Nucl. Phys. B250, 1991985.

[3] G. Sterman, Phys. Lett. B 179, 281 (1986); Nucl. Phys. B281, 310 (1987).

[4] S. Catani and L. Trentadue, Nucl. Phys. B327, 323 (1989); Nucl. Phys. B353, 183 (1991).

[5] G.P. Korchemsky and G. Marchesini, Nucl. Phys. B406, 225 (1993); Phys. Lett. B 313, 433 (1993).

[6] H-n. Li, Phys. Lett. B454, 328 (1999).

[7] E. Laenen, G. Sterman, and W. Vogelsang, Phys. Rev. Lett. 84, 4296 (2000); Phys. Rev. D 63, 114018 (2001).

[8] V.N. Gribov and L.N. Lipatov, Sov. J. Nucl. Phys. 15, 438 (1972); G. Altarelli and G. Parisi, Nucl. Phys. B126, 298 (1977); Yu.L. Dokshitzer, Sov. Phys. JETP 46, 641 (1977).

[9] E.A. Kuraev, L.N. Lipatov and V.S. Fadin, Sov. Phys. JETP 45, 199 (1977); Ya.Ya. Balitsky and L.N. Lipatov, Sov. J. Nucl. Phys. 28, 822 (1978); L.N. Lipatov, Sov. Phys. JETP 63, 904 (1986).

[10] M. Ciafaloni, Nucl. Phys. B296, 49 (1988); S. Catani, F. Fiorani, and G. Marchesini, Phys. Lett. B 234, 339 (1990); Nucl. Phys. B336, 18 (1990); G. Marchesini, Nucl. Phys. B445, 49 (1995).

[11] , H-n. Li, Phys. Rev. D 53, 4970 (1996).

[12] H-n. Li, Z. Li, Zhao, and C.-P. Yuan, Phys. Rev. Lett. 107, 152001 (2011); Phys. Rev. D 87, 074025 (2013).

[13] T. Aaltonen et al. (CDF Collaboration), Phys. Rev. D 85, 091101 (2012).

[14] D.E. Acosta et al. (CDF Collaboration), Phys. Rev. D 71, 112002 (2005).

[15] CMS Collaboration, Report CMS-PAS-QCD-10-014 (2010).

[16] H-n. Li and H.L. Yu, Phys. Rev. Lett. 74, 4388 (1995); Phys. Lett. B 353, 301 (1995); Phys. Rev. D 53, 2480 (1996).

[17] G.P. Korchemsky and G. Sterman, Phys. Lett. B 340, 96 (1994).

[18] H-n. Li, Phys. Lett. B369, 137 (1996); Phys. Rev. D 55, 105 (1997).

[19] J. Botts and G. Sterman, Nucl. Phys. B325, 62 (1989).

[20] H-n. Li and G. Sterman, Nucl. Phys. B381, 129 (1992); H-n. Li, Phys. Rev. D 48, 4243 (1993).

[21] C. Corianó and H-n. Li, Phys. Lett. B309, 409 (1993); Nucl. Phys. B434, 535 (1995).

[22] H-n. Li and H.L. Yu, Phys. Rev. Lett. 74, 4388 (1995); Phys. Lett. B353, 301 (1995); H-n. Li, Phys. Lett. B348, 597 (1995).

[23] H-n. Li, Phys. Rev. D52, 3958 (1995); C.Y. Wu, T.W. Yeh and H-n. Li, Phys. Rev. D 53, 4982 (1996).

[24] J.C. Collins, Adv. Ser. Direct. High Energy Phys. 5, 573 (1989).

[25] X. Ji, and F. Yuan, Phys. Lett. B 543, 66 (2002); A.V. Belitsky, X. Ji, and F. Yuan, Nucl. Phys. B656, 165 (2003).

[26] I.O. Cherednikov and N.G. Stefanis, Nucl. Phys. B802, 146 (2008).

[27] J. Kodaira and L. Trentadue, Phys. Lett. B 112, 66 (1982).

[28] H.L. Lai and H.-n. Li, Phys. Rev. D 58, 114020 (1998).

[29] H.L. Lai et al., Phys. Rev. D 55, 1280 (1997).

[30] T. Becher, C. Lorentzen, and M.D. Schwartz, Phys. Rev. D 86, 054026 (2012).

[31] H-n. Li, Chin. J. Phys. 37, 8 (1999); Phys. Lett. B 405, 347 (1997).

[32] J. Kwieciński, A.D. Martin, and P.J. Sutton, Phys. Rev. D 53, 6094 (1996).

[33] M. Froissart, Phys. Rev. 123, 1053 (1961).

[34] V.S. Fadin and L.N. Lipatov, Phys. Lett. B 429, 127 (1998); G. Camici and M. Ciafaloni, Phys. Lett. B 430, 349 (1998).

[35] R.S. Thorne, Phys. Rev. D 60, 054031 (1999).

[36] H.L. Lai and H.-n. Li, Phys. Lett. B 471, 220 (1999).

[37] M. Beneke, P. Falgari, S. Klein, and C. Schwinn, Nucl. Phys. B855, 695 (2012).

[38] R. Rajkhowa and J.K. Sarma, arXiv:1209.4350 [hep-ph]. 
[39] A.D. Martin, R.G. Roberts, W.J. Stirling, and R.S. Thorne, Eur. Phys. J. C 23, 73 (2002).

[40] G. Bozzi, B. Fuks, and M. Klasen, Nucl. Phys. B794, 46 (2008).

[41] G. Sterman and S. Weinberg, Phys. Rev. Lett. 39, 1436 (1977).

[42] G. Sterman, arXiv:hep-ph/9606312.

[43] L.G. Almeida et al., Phys. Rev. D 79, 074012 (2009).

[44] G. Soyez, G.P. Salam, J. Kim, S. Dutta, and M. Cacciari, arXiv:1211.2811 [hep-ph].

[45] J. Pumplin et al., JHEP 07, 012 (2002).

[46] W. Skiba and D. Tucker-Smith, Phys. Rev. D 75, 115010 (2007).

[47] B. Holdom, JHEP 08, 069 (2007).

[48] L.G. Almeida et al., Phys. Rev. D 79, 074017 (2009).

[49] A. Altheimer et al., J. Phys. G 39, 063001 (2012).

[50] M.H. Seymour, Nucl.Phys. B513, 269 (1998).

[51] S.D. Ellis et al., JHEP 11, 101 (2010).

[52] R. Kelley, M.D. Schwartz, and H.X. Zhu, arXiv: 1102.0561 [hep-ph].

[53] R. Kelley, M.D. Schwartz, R.M. Schabinger, and H.X. Zhu, Phys. Rev. D 86, 054017 (2012).

[54] M. Cacciari, G.P. Salam, and G. Soyez, JHEP 04, 063 (2008). 